\title{
Exact Partition Functions for Potts Antiferromagnets on Cyclic Lattice Strips
}

\author{
Robert $\operatorname{Shrock}^{(a) *}$, Shan-Ho Tsai ${ }^{(b) \dagger}$ \\ (a) Institute for Theoretical Physics \\ State University of New York \\ Stony Brook, N. Y. 11794-3840 \\ (b) Department of Physics and Astronomy \\ University of Georgia \\ Athens, GA 30602
}

\begin{abstract}
We present exact calculations of the zero-temperature partition function of the $q$-state Potts antiferromagnet on arbitrarily long strips of the square, triangular, and kagomé lattices with width $L_{y}=2$ or 3 vertices and with periodic longitudinal boundary conditions. From these, in the limit of infinite length, we obtain the exact ground-state entropy $S_{0}=k_{B} \ln W$. These results are of interest since this model exhibits nonzero ground state entropy $S_{0}>0$ for sufficiently large $q$ and hence is an exception to the third law of thermodynamics. We also include results for homeomorphic expansions of the square lattice strip. The analytic properties of $W(q)$ are determined and related to zeros of the chromatic polynomial for long finite strips.

05.20.-y, 64.60.C, 75.10.H
\end{abstract}

*email: robert.shrock@sunysb.edu

†email: tsai@hal.physast.uga.edu 


\section{INTRODUCTION}

The $q$-state Potts antiferromagnet (AF) [1,2] exhibits nonzero ground state entropy, $S_{0}>$ 0 (without frustration) for sufficiently large $q$ on a given graph or lattice. This is equivalent to a ground state degeneracy per site $W>1$, since $S_{0}=k_{B} \ln W$. Such nonzero ground state entropy is important as an exception to the third law of thermodynamics [3, [4]. The zero-temperature partition function of the above-mentioned $q$-state Potts antiferromagnet on a graph $G$ satisfies

$$
Z(G, q, T=0)_{P A F}=P(G, q)
$$

where $P(G, q)$ is the chromatic polynomial expressing the number of ways of coloring the vertices of the graph $G$ with $q$ colors such that no two adjacent vertices have the same color [5].6]. Thus, formally,

$$
W(\{G\}, q)=\lim _{n \rightarrow \infty} P(G, q)^{1 / n}
$$

where $n=v(G)$ is the number of vertices of $G$, and we use the notation

$$
\{G\}=\lim _{n \rightarrow \infty} G
$$

The minimum number of colors needed for this coloring of $G$ is called its chromatic number, $\chi(G)$. At certain special points $q_{s}$ (typically $q_{s}=0,1, . ., \chi(G)$ ), one has the noncommutativity of limits

$$
\lim _{q \rightarrow q_{s}} \lim _{n \rightarrow \infty} P(G, q)^{1 / n} \neq \lim _{n \rightarrow \infty} \lim _{q \rightarrow q_{s}} P(G, q)^{1 / n}
$$

and hence it is necessary to specify the order of the limits in the definition of $W\left(\{G\}, q_{s}\right)[7]$. As in []], we shall use the first order of limits here; this has the advantage of removing certain isolated discontinuities that could be present in the $W$ with the opposite order of limits. In addition to Refs. [2]- [7], some other previous related works include Refs. [8]- [24]. Since $P(G, q)$ is a polynomial, one can generalize $q$ from $\mathbb{Z}_{+}$to $\mathbb{R}$ and indeed to $\mathbb{C}$. $W(\{G\}, q)$ is a real analytic function for real $q$ down to a minimum value, $q_{c}(\{G\})[7,14$. For a given $\{G\}$, we denote the continuous locus of non-analyticities of $W$ as $\mathcal{B}$. This locus $\mathcal{B}$ forms as the accumulation set of the zeros of $P(G, q)$ (called the chromatic zeros of $G$ ) as $n \rightarrow \infty$ and satisfies $\mathcal{B}(q)=\mathcal{B}\left(q^{*}\right)$. A fundamental question concerning the Potts antiferromagnet is the form of this locus for a given graph family or lattice and, in particular, the maximal region in the complex $q$ plane to which one can analytically continue the function $W(\{G\}, q)$ from physical values where there is nonzero ground state entropy, i.e., $W>1$. We denote this 
region as $R_{1}$. Further, we denote as $q_{c}$ the maximal point where $\mathcal{B}$ intersects the real axis, which can occur via $\mathcal{B}$ crossing this axis or via a line segment of $\mathcal{B}$ lying along this axis.

Although $W(\{G\}, q)$ has been calculated exactly for the triangular lattice [11] and a number of different families of graphs [7], [15]- [23], it has never been calculated exactly (as a general function of $q$ ) for any other 2D (or higher-dimensional) lattice. Accordingly, one useful procedure is to calculate $W$ exactly on infinite-length, finite-width strips of various $2 \mathrm{D}$ lattices. As the width increases, the values of the resulting $W$ functions approach those of the $W$ functions on the respective 2D lattices. We have carried out this procedure for strips with free boundary conditions in the longitudinal direction (in which the length goes to infinity) and free and periodic boundary conditions in the transverse direction [19,22]. For a given lattice, the calculation of $P$ and $W$ on a cyclic strip of a lattice, i.e a strip with periodic boundary conditions in the longitudinal direction (and some choice of boundary conditions in the transverse direction) is more difficult and the results more complicated than is the case for the corresponding strip with free longitudinal boundary conditions (and the same transverse boundary conditions). Despite the greater difficulty, the calculations of $W$ on cyclic strip graphs are worth the effort, because, as explained further below, the resultant $W$ functions exhibit, for finite $L_{y}$, certain analytic features expected for the $W$ functions on the infinite 2D lattice, whereas for strips with free longitudinal boundary conditions, these features are absent (approached in the limit $L_{y} \rightarrow \infty$ ).

Accordingly, we have carried out exact calculations of the chromatic polynomials $P$ and, in the infinite-length limit, the resultant functions $W$, for cyclic strips of the square and kagomé lattices, and we report the results here. $\downarrow$ Since the $W$ functions that we have computed for these strips exhibit the analytic properties expected for the $W$ functions on the respective $2 \mathrm{D}$ lattices, they constitute, in this sense, the closest exact results that one has to these $W$ functions. We also include results for some other families of cyclic strips. For all of these strips the boundary conditions in the transverse direction are free.

From our earlier exact calculations of $W$ on a number of families of graphs we have inferred several general results on $\mathcal{B}$ : (i) for a graph $G$ with well-defined lattice structure, a sufficient condition for $\mathcal{B}$ to separate the $q$ plane into different regions is that $G$ contains at least one global circuit, defined as a route following a lattice direction which has the topology of $S^{1}$ and a length $\ell_{\text {g.c. }}$ that goes to infinity as $n \rightarrow \infty$. 19

\footnotetext{
${ }^{1} \mathrm{~A}$ brief report of some of the results for $W$ is given in Ref. [25].

${ }^{2}$ Some families of graphs that do not have regular lattice directions have noncompact loci $\mathcal{B}$ that separate
} 
For a $d$-dimensional lattice graph, the existence of global circuits is equivalent to having periodic boundary conditions (BC's) in at least one direction. Further, (ii) the general condition for a family $\{G\}$ to have a locus $\mathcal{B}$ that is noncompact (unbounded) in the $q$ plane [16] shows that a sufficient (not necessary) condition for $\{G\}$ to have a compact, bounded locus $\mathcal{B}$ is that it is a regular lattice [16,18,23]. The third and fourth general features are that for graphs that (a) contain global circuits, (b) cannot be written in the form $G=K_{p}+H$ [15], and (c) have compact $\mathcal{B}$, we have observed that $\mathcal{B}$ (iii) passes through $q=0$ and (iv) crosses the positive real axis, thereby always defining a $q_{c}$. As noted, the advantage of cyclic strip graphs is that these properties are present for each finite $L_{y}$ rather than only being approached in the limit $L_{y} \rightarrow \infty$ as for open strips.

Our present results also strengthen the evidence for our earlier conjectures [19,23] that on a graph with well-defined lattice structure, a necessary property for there to be chromatic zeros and, in the $n \rightarrow \infty$ limit, a locus $\mathcal{B}$ including support for $\operatorname{Re}(q)<0$, is that the graph has at least one global circuit. (This is known not to be a sufficient property, as shown, e.g., by the circuit and ladder graphs, which have such global circuits, but whose chromatic zeros and loci $\mathcal{B}$ have support only for $\operatorname{Re}(q) \geq 0$.)

\section{GENERAL PROPERTIES AND CALCULATIONAL METHOD}

A general form for the chromatic polynomial of an $n$-vertex strip graph $G$ of the type considered here (and more generally, a graph built up recursively by the successive addition of a given subgraph) is

$$
P(G, q)=\sum_{j=1}^{N_{a}} c_{j}(q)\left(a_{j}(q)\right)^{t_{j} n}
$$

the $q$ plane into different regions [16, 18,23].

\footnotetext{
${ }^{3}$ The complete graph on $p$ vertices, denoted $K_{p}$, is the graph in which every vertex is adjacent to every other vertex. The "join" of graphs $G_{1}$ and $G_{2}$, denoted $G_{1}+G_{2}$, is defined by adding bonds linking each vertex of $G_{1}$ to each vertex in $G_{2}$. Graph families with $\mathcal{B}$ not including $q=0$ are given in [15, 16, 18,23$]$.
} 
where $c_{j}(q)$ and $a_{j}(q)$ are certain functions of $q$. Here the $a_{j}(q)$ and $c_{j}(q)$ are independent of $n$, and $t_{j}$ is a $G$-dependent constant. As before [7], we define a term $a_{\ell}(q)$ as "leading" $(\ell)$ if it dominates the $n \rightarrow \infty$ limit of $P(G, q)$; in particular, if $N_{a} \geq 2$, then it satisfies $\left|a_{\ell}(q)\right|>\left|a_{j}(q)\right|$ for $j \neq \ell$, so that $|W|=\left|a_{\ell}\right|^{t_{\ell}}$. The locus $\mathcal{B}$ occurs where there is a nonanalytic change in $W$ as the leading terms $a_{\ell}$ in eq. (2.1) changes. Note that a term of the form $a_{1}= \pm 1$ may be absent. For some families of graphs, the $c_{j}(q)$ and $a_{j}(q)$ are polynomials. However, there are also many families of graphs for which $c_{j}(q)$ and $a_{j}(q)$ are not polynomial, but instead, are algebraic, functions of $q$; for these families the property that the chromatic polynomial is, in fact, a polynomial of $q$, is not manifest from the expression (2.1).

For a strip graph $G_{s}$ our calculational method is to use the deletion-contraction theorem [5] iteratively to obtain a set of linear equations which we then solve, to get a generating function $\Gamma\left(G_{s}, q, x\right)$; the chromatic polynomials for the strip of length $L_{x}=m$ are then determined as the coefficients in a Taylor series expansion of this generating function in an auxiliary variable $x$ about $x=0$ :

$$
\Gamma\left(G_{s}, q, x\right)=\sum_{m=m_{0}}^{\infty} P\left(\left(G_{s}\right)_{m}, q\right) x^{m-m_{0}}
$$

where $m_{0}$ depends on the type of strip graph $G_{s}$ and is naturally chosen as the minimal value of $m$ for which the graph is well defined. The generating functions $\Gamma\left(G_{s}, q, x\right)$ are rational functions of the form

$$
\Gamma\left(G_{s}, q, x\right)=\frac{\mathcal{N}\left(G_{s}, q, x\right)}{\mathcal{D}\left(G_{s}, q, x\right)}
$$

with

$$
\mathcal{N}\left(G_{s}, q, x\right)=\sum_{j=0}^{d_{\mathcal{N}}} A_{G_{s}, j}(q) x^{j}
$$

and

\footnotetext{
${ }^{4}$ The labelling convention for the summation index $j$ in eq. (2.1) is the same as that in our Ref. [21]; this differs from the convention we used in Ref. [7], where $j$ started at 0 , and where the term denoted here as $c_{1}\left(a_{1}\right)^{t_{1} n}$ was labelled simply as $c_{0}$ since $c_{0}$ was either 1 or proportional to $(-1)^{n}$, and hence did not grow with $n$ like (const. $)^{n}$ with $\mid$ const. $\mid>1$.
} 


$$
\mathcal{D}\left(G_{s}, q, x\right)=1+\sum_{j=1}^{d_{\mathcal{D}}} b_{G_{s}, j}(q) x^{j}
$$

where the $A_{G_{s}, i}$ and $b_{G_{s}, i}$ are polynomials in $q$ (with no common factors) f, and the degrees of the numerator and denominator, as polynomials in the auxiliary variable $x$, are

$$
d_{\mathcal{N}}=\operatorname{deg}_{x}(\mathcal{N})
$$

and

$$
d_{\mathcal{D}}=\operatorname{deg}_{x}(\mathcal{D})
$$

Writing the denominator of the generating function $\Gamma\left(G_{s}, q, x\right)$ in factorized form, we have

$$
\mathcal{D}\left(G_{s}, q, x\right)=\prod_{j=1}^{d_{\mathcal{D}}}\left(1-\lambda_{G_{s}, j}(q) x\right)
$$

(c.f. eq. (4.2) in Ref. [19] or (2.9) in Ref. [21]). The chromatic polynomials can equivalently be calculated from a corresponding recurrence relation (see eq. (2.25) in Ref. [21]).

We define the polynomial

$$
D_{k}(q)=\frac{P\left(C_{k}, q\right)}{q(q-1)}=a^{k-2} \sum_{j=0}^{k-2}(-a)^{-j}=\sum_{s=0}^{k-2}(-1)^{s}\left(\begin{array}{c}
k-1 \\
s
\end{array}\right) q^{k-2-s}
$$

where $a=q-1$ and $P\left(C_{k}, q\right)$ is the well-known chromatic polynomial for the circuit (cyclic) graph $C_{k}$ with $k$ vertices,

$$
P\left(C_{k}, q\right)=a^{k}+(-1)^{k} a
$$

In Table 1 we list the values of $d_{\mathcal{N}}$ and $d_{\mathcal{D}}$ for various families of lattice strip graphs considered in this paper and in our previous ones. The form of $\mathcal{N}$ and value of $d_{\mathcal{N}}$, (but not $\mathcal{D}$ or $d_{\mathcal{D}}$ ) depend on one's convention for $m_{0}$ in eq. (2.2); the values given in Table 1 correspond to the conventions that we use in this paper and our previous ones.

In eq. (2.17) of Ref. 21] we gave a general formula that enables one to calculate the chromatic polynomial in the closed form (2.1) from the generating function or vice versa.

\footnotetext{
${ }^{5}$ As in Ref. 21], we use the notation $A_{G_{s}, i}$ rather than our earlier notation $a_{G_{s}, i}$ in Ref. [19] in order to avoid confusion with the terms $a_{j}$ in the general formula 2.1).
} 
Since the denominators of generating functions for wider strips of various lattices $G_{s}$ yield $\lambda_{G_{s}, j}$ 's in eq. (2.8) that are algebraic, but not polynomial, functions of $q$, it can be more convenient to obtain the chromatic polynomials directly from the expansion of the generating functions. However, for the simplest types of strips, the $\lambda_{G_{s}, j}$ 's are polynomial, and in these cases the closed-form expression (2.1) is the most convenient. In the case of cyclic strips of the square lattice, it is worthwhile to give the generating functions for the simpler cases $L_{y}=1$ (the circuit graph) and $L_{y}=2$ (the cyclic ladder graph) as a comparison with the much more complicated results for the $L_{y}=3$ case that we are reporting here. For these cyclic strips one can write

$$
\Gamma\left(s q\left(L_{y}, c y c .\right), q, x\right)=\sum_{m=2}^{\infty} P\left(s q\left(L_{y}, c y c .\right)_{m}, q\right) x^{m-2}
$$

As indicated, it is natural to take $m_{0}=2$ for the cyclic strip of the square lattice (and for the cyclic strip of the triangular lattice to be discussed later) because the lowest value of $m$ is $m=2$, for which the respective strips reduce to a single column of squares or triangles. For the minimal case, $L_{y}=1$, the generating function that yields, as the $x^{m-2}$ coefficient, the chromatic polynomial $P\left(C_{m}, q\right)$, is

$$
\Gamma\left(s q\left(L_{y}=1, c y c .\right), q, x\right)=\frac{q(q-1)}{[1-(q-1) x](1+x)}
$$

As an example of the $m_{0}$-dependence of $\mathcal{N}$, we note that if one used a definition with $m_{0}=0$, i.e., $\tilde{\Gamma}\left(s q\left(L_{y}, c y c\right.\right.$. $\left.), q, x\right)=\sum_{m=0}^{\infty} P\left(s q\left(L_{y}, c y c .\right)_{m}, q\right) x^{m}$, (where the $m=0,1$ coefficients are only formal) then

$$
\begin{aligned}
\tilde{\Gamma}\left(s q\left(L_{y}=1, c y c .\right), q, x\right) & =\frac{q[1-(q-2) x]}{[1-(q-1) x](1+x)}=\frac{1}{1-(q-1) x}+\frac{q-1}{1-x} \\
& =\sum_{m=0}^{\infty}\left[(q-1)^{m}+(q-1)(-1)^{m}\right] x^{m}
\end{aligned}
$$

which, of course, agrees with the expression for $P\left(s q\left(L_{y}=1\right)_{m}, q\right) \equiv P\left(C_{m}, q\right)$ given in eq. (2.10). For $L_{y}=2$, from the known chromatic polynomial [9], we determine the corresponding generating function for eq. (2.11) (with the convention $m_{0}=2$ ) to be

$$
\Gamma\left(s q\left(L_{y}=2, c y c .\right), q, x\right)=\frac{q(q-1)\left[D_{4}-2(q-1) x-(q-1)(q-3) D_{4} x^{2}\right]}{\left(1-D_{4} x\right)(1-x)[1-(1-q) x][1-(3-q) x]}
$$

where $D_{4}=q^{2}-3 q+3$. As will be seen from our new results, the complexity of the generating function increases considerably when one goes from width $L_{y}=2$ to $L_{y}=3$.

TABLE I. Properties of generating functions for strip graphs $G_{s}$. 


\begin{tabular}{ccc}
\hline \hline$G_{s}$ & $\operatorname{deg}_{x}(\mathcal{N})$ & $\operatorname{deg}(\mathcal{D})$ \\
\hline$s q\left(L_{y}=1, F B C_{x}\right)$ & 0 & 1 \\
$s q\left(L_{y}=2, F B C_{x}\right)$ & 0 & 1 \\
$s q\left(L_{y}=3, F B C_{x}\right)$ & 1 & 2 \\
$s q\left(L_{y}=4, F B C_{x}\right)$ & 2 & 3 \\
\hline$s q\left(L_{y}=1, P B C_{x}\right)$ & 0 & 2 \\
$s q\left(L_{y}=2, P B C_{x}\right)$ & 2 & 4 \\
$s q\left(L_{y}=3, P B C_{x}\right)$ & 8 & 10 \\
\hline$t\left(L_{y}=2, F B C_{x}\right)$ & 0 & 1 \\
$t\left(L_{y}=3, F B C_{x}\right)$ & 1 & 2 \\
$t\left(L_{y}=4, F B C_{x}\right)$ & 3 & 4 \\
\hline$t\left(L_{y}=2, P B C_{x}\right)$ & 2 & 4 \\
\hline$k g\left(L_{y}=2, F B C_{x}\right)$ & 1 & 2 \\
\hline$k g\left(L_{y}=2, P B C_{x}\right)$ & 8 & 9 \\
\hline \hline
\end{tabular}


For a given $G_{s}$, in region $R_{1}$, the function $W$ is given by

$$
W=\left(\lambda_{\max }\right)^{t}
$$

For $q$ in regions other than $R_{1}$, one can only determine the magnitude $|W(\{G\}, q)|$ unambiguously [7]; this is given by

$$
|W|=\left|\lambda_{\max }\right|^{t}
$$

where $\lambda_{\max }$ denotes the $\lambda$ with maximal magnitude in the respective region and

$$
t=\frac{m}{n}
$$

For the square and triangular strips, $t=1 / L_{y}$ while for the kagomé strip considered here, $t=1 / 5$. Thus, $W$ and $\mathcal{B}$ are determined by $\mathcal{D}$, independent of $\mathcal{N}$. Of course, the determination of $\mathcal{N}$ is necessary to calculate the actual chromatic polynomials for particular finite-length strips. Besides their intrinsic interest in the context of mathematical graph theory, these chromatic polynomials for finite strips are important because from them one can calculate their zeros and thereby check how rapidly these approach the asymptotic $n \rightarrow \infty$ accumulation set $\mathcal{B}$. (Indeed, for some other families of graphs, we have shown that the chromatic zeros lie precisely on the asymptotic loci even for finite $n$ [15].)

The organization of the paper is as follows. Our calculations for the strips of the square, kagomé, and triangular lattices are given in sections 3-5 (see also the appendix). In section 6 we include results for certain homeomorphic expansions of square strips. Our concluding remarks are given in section 7 .

\section{CYCLIC STRIP OF THE SQUARE LATTICE WITH $L_{y}=3$}

We consider the cyclic strip of the square (sq) lattice of length $L_{x}=m$ vertices and width $L_{y}=3$ vertices. This has $n=L_{x} L_{y}=3 m$ vertices, $e=5 m$ edges (for the nondegenerate cases $m>2$ ), and chromatic number (for any $L_{y}$ )

$$
\chi\left(s q\left(L_{y}\right)_{m}, c y c .\right)= \begin{cases}2 & \text { for even } m \\ 3 & \text { for odd } m\end{cases}
$$

(For the minimal case $m=2$, the periodic longitudinal boundary conditions lead the strip to degenerate to a single tower of two squares.) We use a generating function of the form

$$
\Gamma\left(s q\left(L_{y}=3, \text { cyc. }\right), q, x\right)=\sum_{m=2}^{\infty} P\left(s q\left(L_{y}=3, c y c .\right)_{m}, q\right) x^{m-2}
$$


where $P\left(s q\left(L_{y}=3, c y c .\right)_{m}, q\right)$ is the chromatic polynomial for the strip of length $m$ vertices. We obtain a generating function of the form (2.3) with (2.4) and (2.5), where $\operatorname{deg}_{x}(\mathcal{N})=$ 8 and $\operatorname{deg}_{x}(\mathcal{D})=10$. Clearly this is considerably more complicated than the generating function for the strip with the same width, $L_{y}=3$, but with free longitudinal boundary conditions (and again free transverse boundary conditions), which had $\operatorname{deg}_{x}(\mathcal{N})=1$ and $\operatorname{deg}_{x}(\mathcal{D})=2$. This and other comparisons are shown in Table 1 .

We have calculated the denominator to be

$$
\begin{aligned}
\mathcal{D}\left(s q\left(L_{y}=3\right), q, x\right)= & {[1+x][1-(q-1) x][1-(q-2) x][1-(q-4) x]\left[1+(q-2)^{2} x\right] } \\
& \times\left[1+b_{s q, 11} x+b_{s q, 12} x^{2}\right]\left[1+b_{s q, 21} x+b_{s q, 22} x^{2}+b_{s q, 23} x^{3}\right]
\end{aligned}
$$

where

$$
\begin{gathered}
b_{s q, 11}=-(q-2)\left(q^{2}-3 q+5\right) \\
b_{s q, 12}=(q-1)\left(q^{3}-6 q^{2}+13 q-11\right) \\
b_{s q, 21}=2 q^{2}-9 q+12 \\
b_{s q, 22}=q^{4}-10 q^{3}+36 q^{2}-56 q+31 \\
b_{s q, 23}=-(q-1)\left(q^{4}-9 q^{3}+29 q^{2}-40 q+22\right)
\end{gathered}
$$

Thus,

$$
\mathcal{D}\left(s q\left(L_{y}=3, \text { cyc. }\right), q, x\right)=\prod_{j=1}^{10}\left(1-\lambda_{s q, j} x\right)
$$

where the first five $\lambda$ 's can be read off immediately from the linear factors in eq. (3.3),

$$
\lambda_{s q,(6,7)} \equiv \lambda_{s q, 1, \pm}=\frac{1}{2}\left[-b_{s q, 11} \pm\left(b_{s q, 11}^{2}-4 b_{s q, 12}\right)^{1 / 2}\right]
$$

and $\lambda_{s q, j}, j=8,9,10$ are the roots of the cubic

$$
\xi^{3}+b_{s q, 21} \xi^{2}+b_{s q, 22} \xi+b_{s q, 23}=0
$$

From this we have calculated the locus $\mathcal{B}$, shown in Fig. 11. This locus separates the $q$ plane into seven regions: $R_{1}$ including the real interval $q>q_{c} ; R_{2}$, to the left of $R_{1} ; R_{3}$ 
including the real interval $0<q<2$; two complex-conjugate (c.c.) phases $R_{4}, R_{4}^{*}$ centered at $q \simeq 2.4 \pm 0.9 i$; and two additional quite narrow, sliver-like c.c. phases $R_{5}, R_{5}^{*}$ centered at $q \simeq 1.95 \pm 1.75 i$. In region $R_{1}$, the dominant $\lambda$ is $\lambda_{s q, 6} \equiv \lambda_{s q, 1,+}$, so that

$$
\begin{aligned}
W\left(s q\left(L_{y}=3, \text { cyc. }\right), q\right)= & 2^{-1 / 3}\left[(q-2)\left(q^{2}-3 q+5\right)+\right. \\
& {\left.\left[\left(q^{2}-5 q+7\right)\left(q^{4}-5 q^{3}+11 q^{2}-12 q+8\right)\right]^{1 / 2}\right]^{1 / 3} \quad \text { for } \quad q \in R_{1} }
\end{aligned}
$$

In region $R_{2}$, the dominant $\lambda$ is $q-4$, so

$$
\mid W\left(s q\left(L_{y}=3, \text { cyc. }\right), q\right)|=| q-\left.4\right|^{1 / 3} \quad \text { for } \quad q \in R_{2}
$$

The value of $q_{c}$ for this strip is given as the real solution to the equation $\left|W_{R_{1}}\right|=\left|W_{R_{2}}\right|$, which reduces to

$$
2 q^{4}-16 q^{3}+51 q^{2}-86 q+67=0
$$

yielding

$$
q_{c}\left(s q\left(L_{y}=3, \text { cyc. }\right)\right)=2.33654
$$

At this point $W=1.18487$. In region $R_{3}$, the dominant $\lambda$ is the maximal root of the cubic (3.11). The degeneracy condition $\left|W_{R_{2}}\right|=\left|W_{R_{3}}\right|$ yields the value $q=2$ for the point separating $R_{3}$ and $R_{2}$ on the real axis. Note that the portion of the boundary $\mathcal{B}$ separating regions $R_{2}$ and $R_{3}$ is an arc of the circle $|q-4|=2$. In the c.c. pairs of regions $\left(R_{4}, R_{4}^{*}\right)$ and $\left(R_{5}, R_{5}^{*}\right)$ the dominant $\lambda$ 's are the other two roots of the cubic (3.11). As discussed before, these results depend only on $\mathcal{D}$. As is evident in Fig. 1, $\mathcal{B}$ contains several multiple points, forming conjugate complex pairs. The property that there is no multiple point at $q_{c}$ follows because only two of the $\lambda$ 's become degenerate in magnitude at this point. This may be contrasted with the situation for the $L_{y}=2$ cyclic strip, where three leading terms become degenerate, leading to a multiple point, at $q_{c}\left(s q\left(L_{y}=2, c y c.\right)\right)=2$, at which four curves come together [7]. As noted above, these results depend only on $\mathcal{D}$.

The coefficient functions for the numerator are rather lengthy and hence are given in the Appendix. Together with the above results for $\mathcal{D}$, these yield, via eq. (3.2), the chromatic polynomials for the cyclic strip of the square lattice of arbitrary length. From the generating function, using eq. (2.17) of Ref. [21] (with $m \rightarrow m-1$ to match our current notational conventions), we calculate the chromatic polynomial

$P\left(s q\left(L_{y}=3, \text { cyc. }\right)_{m}, q\right)=\left(q^{3}-5 q^{2}+6 q-1\right)(-1)^{m}$ 


$$
\begin{aligned}
& +\left(q^{2}-3 q+1\right)\left[(q-1)^{m}+(q-2)^{m}+(q-4)^{m}\right]+(q-1)\left[-(q-2)^{2}\right]^{m}+\left[\left(\lambda_{s q, 6}\right)^{m}+\left(\lambda_{s q, 7}\right)^{m}\right] \\
& +(q-1)\left[\left(\lambda_{s q, 8}\right)^{m}+\left(\lambda_{s q, 9}\right)^{m}+\left(\lambda_{s q, 10}\right)^{m}\right]
\end{aligned}
$$

This can also be derived directly from $\mathcal{D}$ without using $\mathcal{N}$ by matching with calculations of $P$ for the first few values of $m$. Thus, in the notation of eq. (2.17) of Ref. [21] or eq. (2.1) here, $a_{s q, j}=\lambda_{s q, j}, c_{s q, 1}=q^{3}-5 q^{2}+6 q-1, c_{s q, j}=q^{2}-3 q+1$ for $j=2,3,4, c_{s q, j}=q-1$ for $j=5,8,9,10$, and $c_{s q, j}=1$ for $j=6,7$. In Fig. 1 we show chromatic zeros for a strip of length $m=20$ and thus $n=60$ vertices, for comparison with the $n \rightarrow \infty$ accumulation set $\mathcal{B}$. Besides the fact that these zeros lie close to $\mathcal{B}$, we observe that the density is largest on the right-hand side of the "main" curve in $\mathcal{B}$ and is rather small for the curve bounding the region $R_{2}$ on the left.

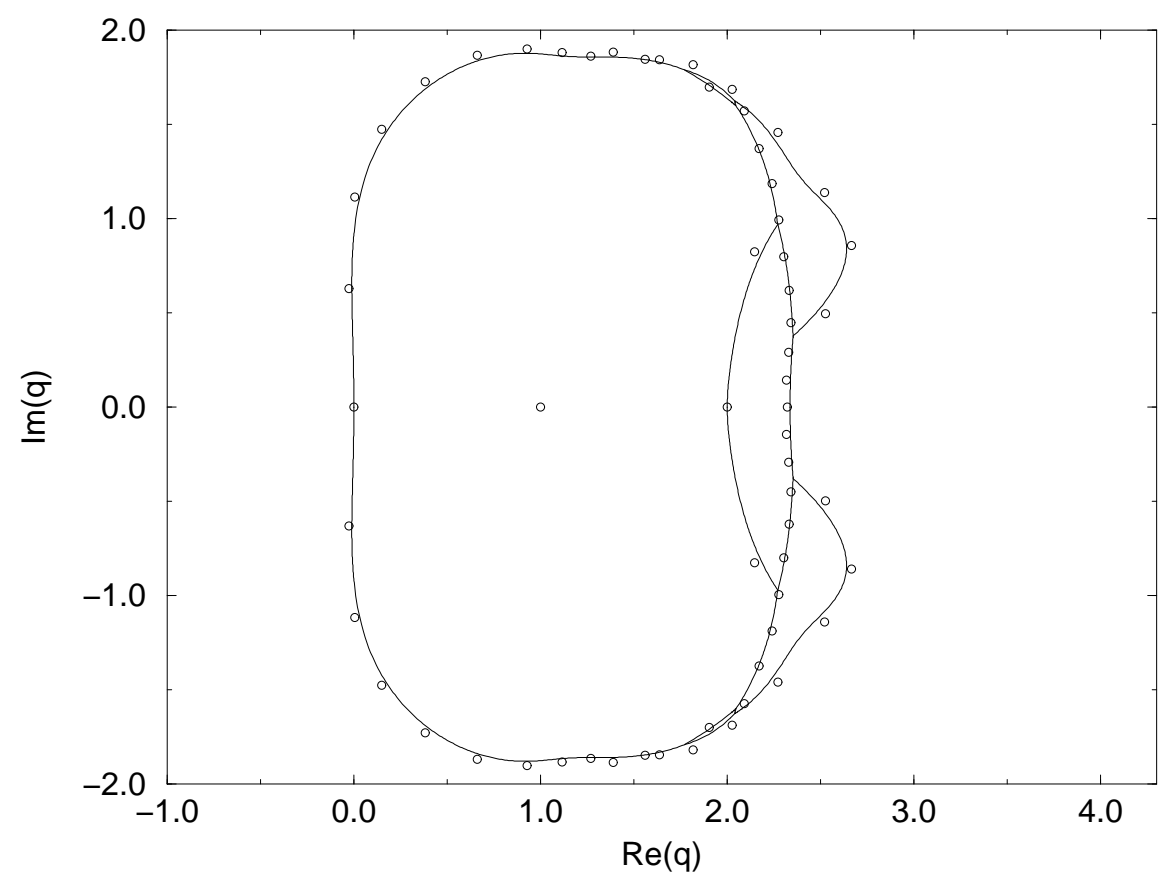

FIG. 1. Boundary $\mathcal{B}$ in the $q$ plane for $W$ function for a cyclic strip of the square lattice with width $L_{y}=3$. Chromatic zeros for $m=20$ and thus $n=60$ vertices are shown for comparison.

An interesting feature of this family of graphs is that the chromatic zeros and their 
accumulation set $\mathcal{B}$ have support for $\operatorname{Re}(q)<0$. Chromatic zeros with negative real parts occur first for $m=10$, i.e., $n=30$.

\section{CYCLIC STRIP OF THE KAGOMÉ LATTICE}

We next consider a cyclic strip of the kagomé lattice comprised of $m$ hexagons with each pair sharing two triangles as adjacent polygons (as shown for the open strip in Fig. 1 in Ref. [19]). This has $n=5 m$ vertices and $e=8 m$ edges. The chromatic number is $\chi=3$. We use a generating function of the form

$$
\Gamma\left(k g\left(L_{y}=2, c y c .\right), q, x\right)=\sum_{m=1}^{\infty} P\left(k g(c y c .)_{m}, q\right) x^{m-1}
$$

where $P\left(k g(c y c .)_{m}, q\right)$ is the chromatic polynomial for the cyclic strip with $m$ hexagons. The generating function $\Gamma$ has $\operatorname{deg}_{x}(\mathcal{N})=8$ and $\operatorname{deg}_{x}(\mathcal{D})=9$, and is thus much more complicated than the generating function for the kagomé strip of the same width with free longitudinal boundary conditions, which had $\operatorname{deg}_{x}(\mathcal{N})=1$ and $\operatorname{deg}_{x}(\mathcal{D})=2$ (see Table 1 ). We have calculated

$$
\begin{gathered}
\mathcal{D}\left(k g\left(L_{y}, c y c .\right), q, x\right)=\left(1+b_{k g, 11} x+b_{k g, 12} x^{2}\right)\left(1+b_{k g, 21} x+b_{k g, 22} x^{2}\right)\left(1+b_{k g, 31} x+b_{k g, 32} x^{2}\right) \times \\
{[1-(q-2) x][1-(q-4) x]\left[1-(q-1)(q-2)^{2} x\right]}
\end{gathered}
$$

where

$$
\begin{gathered}
b_{k g, 11}=-(q-2)\left(q^{4}-6 q^{3}+14 q^{2}-16 q+10\right) \\
b_{k g, 12}=(q-1)^{3}(q-2)^{3} \\
b_{k g, 21}=-q^{3}+7 q^{2}-19 q+20 \\
b_{k g, 22}=(q-1)(q-2)^{3} \\
b_{k g, 31}=11-9 q+2 q^{2} \\
b_{k g, 32}=-(q-1)(q-2)^{2}
\end{gathered}
$$


We thus find, for $1 \leq j \leq 6$,

$$
\begin{gathered}
\lambda_{k g, j} \equiv \lambda_{k g, k, \pm}=\frac{1}{2}\left[-b_{k g, k 1} \pm\left(b_{k g, k 1}^{2}-4 b_{k g, k 2}\right)^{1 / 2}\right], \quad k=1,2,3 \\
\lambda_{k g, 7}=q-2 \\
\lambda_{k g, 8}=q-4 \\
\lambda_{k g, 9}=(q-1)(q-2)^{2}
\end{gathered}
$$

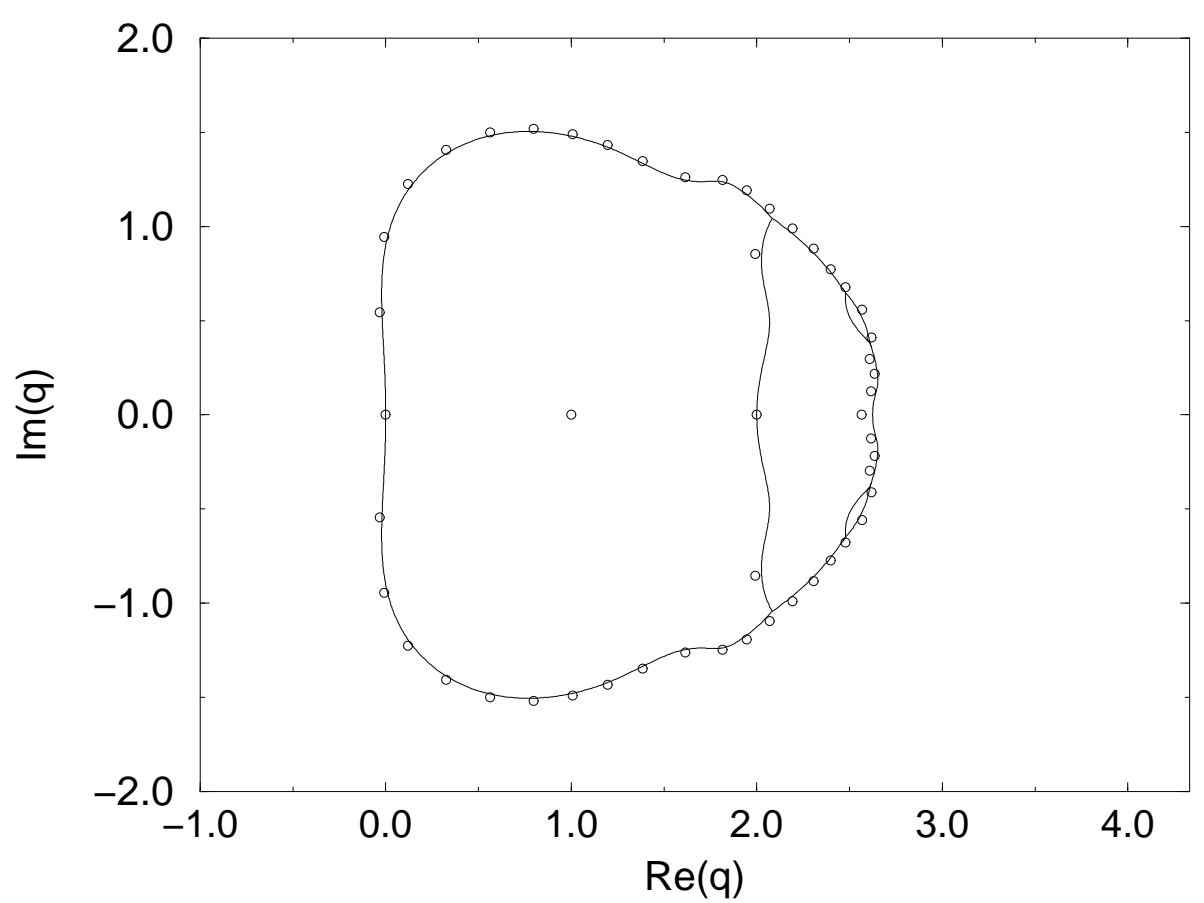

FIG. 2. Locus $\mathcal{B}$ for $W$ for $\infty \times 2$ cyclic strip of the kagomé lattice. Chromatic zeros for $m=10(n=50)$ are also shown for comparison. 
The coefficient functions for the numerator are rather lengthy and hence are given in the Appendix. From the generating function, via eq. (2.17) of Ref. [21], or directly from $\mathcal{D}$ by matching results with computations of $P$ for the first few values of $m$, we calculate the chromatic polynomial

$$
\begin{aligned}
P\left(k g\left(L_{y}=2\right)_{m}, q\right)= & \left(q^{2}-3 q+1\right)(q-4)^{m}+(q-1)\left[(q-1)(q-2)^{2}\right]^{m} \\
& +\left[\left(\lambda_{k g, 1}\right)^{m}+\left(\lambda_{k g, 2}\right)^{m}\right]+(q-1)\left[\left(\lambda_{k g, 3}\right)^{m}+\left(\lambda_{k g, 4}\right)^{m}\right]
\end{aligned}
$$

There are three $\lambda$ 's in $\mathcal{D}$ that do not appear in $P$, namely, $q-2$ and $\lambda_{k g,(5,6)} \equiv \lambda_{k g, 3, \pm}$. In the context of the general formula, eq. (2.17) of Ref. [21], this can be seen as a result of the vanishing of the corresponding coefficient functions $c_{j}$ in eq. (2.19) of that work. Fig. 2 shows the boundary $\mathcal{B}$ in the $q$ plane for the $W$ function. Again, $\mathcal{B}$ divides the $q$ plane into several regions (Fig. 2). In region $R_{1}, W$ is determined by $\lambda_{k g, 1} \equiv \lambda_{k g, 1,+}$ :

$$
\begin{aligned}
W\left(\left\{G_{k g\left(L_{y}=2\right)}\right\}, q\right)= & 2^{-1 / 5}(q-2)^{1 / 5}\left[q^{4}-6 q^{3}+14 q^{2}-16 q+10\right. \\
& \left.+\left[q^{8}-12 q^{7}+64 q^{6}-200 q^{5}+404 q^{4}-548 q^{3}+500 q^{2}-292 q+92\right]^{1 / 2}\right]^{1 / 5}
\end{aligned}
$$

As $q$ decreases through $q_{c},|W|$ switches to $|q-4|^{1 / 5}$; solving the degeneracy equation $\left|\lambda_{k g, 1}\right|=$ $|4-q|$ yields $q_{c}=2.62421$ (at which point $W=1.06588$ ). This is within about $10 \%$ of the inferred exact value $q_{c}=3$ for the $2 \mathrm{D}$ kagomé lattice [22]. It is impressive that an infinite strip of width $L_{y}=2$ yields a $q_{c}$ this close to the value for the full $2 \mathrm{D}$ lattice. In the regions including the intervals $2<q<q_{c}$ and $0<q<2,|W|=|q-4|^{1 / 5}$ and $|W|=\left|\lambda_{k g, 4}\right|^{1 / 5}$, respectively. It is interesting to observe that the boundary $\mathcal{B}$ includes a part with $\operatorname{Re}(q)<0$. Of the nine coefficient functions in the polynomial in $x$ in $\mathcal{D}$, the two that are equal to the coefficient functions for the open strip of the kagomé lattice of the same width are $b_{k g, 11}$ and $b_{k g, 12}$. Thus, the root $\lambda_{k g, 1}$ is leading in region $R_{1}$ for both open and cyclic longitudinal boundary conditions.

To compare with the asymptotic locus $\mathcal{B}$, we have calculated the zeros of the chromatic polynomial for a finite strip with $m=10$, i.e., $n=50$ vertices; these are displayed in Fig. (2). As in the case of the $L_{y}=3$ cyclic strip of the square graph, this cyclic strip of the kagomé lattice has the property that the chromatic zeros and their accumulation set $\mathcal{B}$ have support for $\operatorname{Re}(q)<0$. Chromatic zeros with negative real parts occur first for $m=5$, i.e., $n=25)$. 


\section{CYCLIC STRIP OF THE TRIANGULAR LATTICE WITH $L_{y}=2$}

In this section we consider the cyclic strip of the triangular $(t)$ lattice of length $L_{x}=$ $m$ vertices and width $L_{y}=2$ vertices. This has $n=L_{x} L_{y}=2 m$ vertices and, for the nondegenerate cases $m \geq 3, e=4 m$ edges (bonds). We consider both the cases of periodic and twisted periodic longitudinal boundary conditions, and we denote the correponding strips as cyclic and twisted cyclic (= Möbius, i.e., opposite ends are identified with reversed orientation). For $m=2$ the cyclic and twisted cyclic strips both degenerate to the complete graph on 4 vertices, $K_{4}$, with $e=6$ edges. The cyclic strip has chromatic number

$$
\chi\left(t\left(L_{y}=2, L_{x}=m\right), c y c .\right)= \begin{cases}3 & \text { if } m=0 \bmod 3 \\ 4 & \text { if } m=1 \text { or } m=2 \bmod 3\end{cases}
$$

whereas the twisted cyclic strip has

$$
\chi\left(t\left(L_{y}=2, L_{x}=m\right), \text { twisted cyc. }\right)=4
$$

For these cyclic strips we use generating functions of the form

$$
\begin{gathered}
\Gamma\left(t\left(L_{y}=2, \text { cyc. }\right), q, x\right)=\sum_{m=2}^{\infty} P\left(t\left(L_{y}, c y c .\right)_{m}, q\right) x^{m-2} \\
\Gamma\left(t\left(L_{y}=2, \text { twisted cyc. }\right), q, x\right)=\sum_{m=2}^{\infty} P\left(t\left(L_{y}, \text { twisted cyc. }\right)_{m}, q\right) x^{m-2}
\end{gathered}
$$

where $P\left(t\left(L_{y}, c y c .\right)_{m}, q\right)$ and $P\left(t\left(L_{y}, \text { twisted cyc. }\right)_{m}, q\right)$ are the chromatic polynomials for the respective strips. For these strips, we find that $\operatorname{deg}_{x}(\mathcal{D})=4$ (see Table 1 ). The degrees in $x$ of the numerator for the cyclic and twisted cyclic strips are, respectively, $\operatorname{deg}_{x}(\mathcal{N})=2$ and $\operatorname{deg}_{x}(\mathcal{N})=0$. We calculate

$$
\begin{gathered}
b_{t, 1}=-q^{2}+6 q-10 \\
b_{t, 2}=-(q-3)\left(2 q^{2}-9 q+11\right) \\
b_{t, 3}=-(q-2)^{2}\left(q^{2}-6 q+10\right) \\
b_{t, 4}=(q-2)^{4}
\end{gathered}
$$


The denominator of the generating function has the factorized form

$$
\begin{aligned}
\mathcal{D}\left(t\left(L_{y}=2, c y c .\right), q, x\right)= & (1-x)\left[1-(q-2)^{2} x\right]\left[1+(2 q-5) x+(q-2)^{2} x^{2}\right] \\
& =\prod_{j=1}^{4}\left(1-\lambda_{t, j} x\right)
\end{aligned}
$$

where

$$
\begin{gathered}
\lambda_{t, 1}=1 \\
\lambda_{t, 2}=(q-2)^{2} \\
\lambda_{t, 3}=\frac{1}{2}[5-2 q+\sqrt{9-4 q}] \\
\lambda_{t, 4}=\frac{1}{2}[5-2 q-\sqrt{9-4 q}]
\end{gathered}
$$

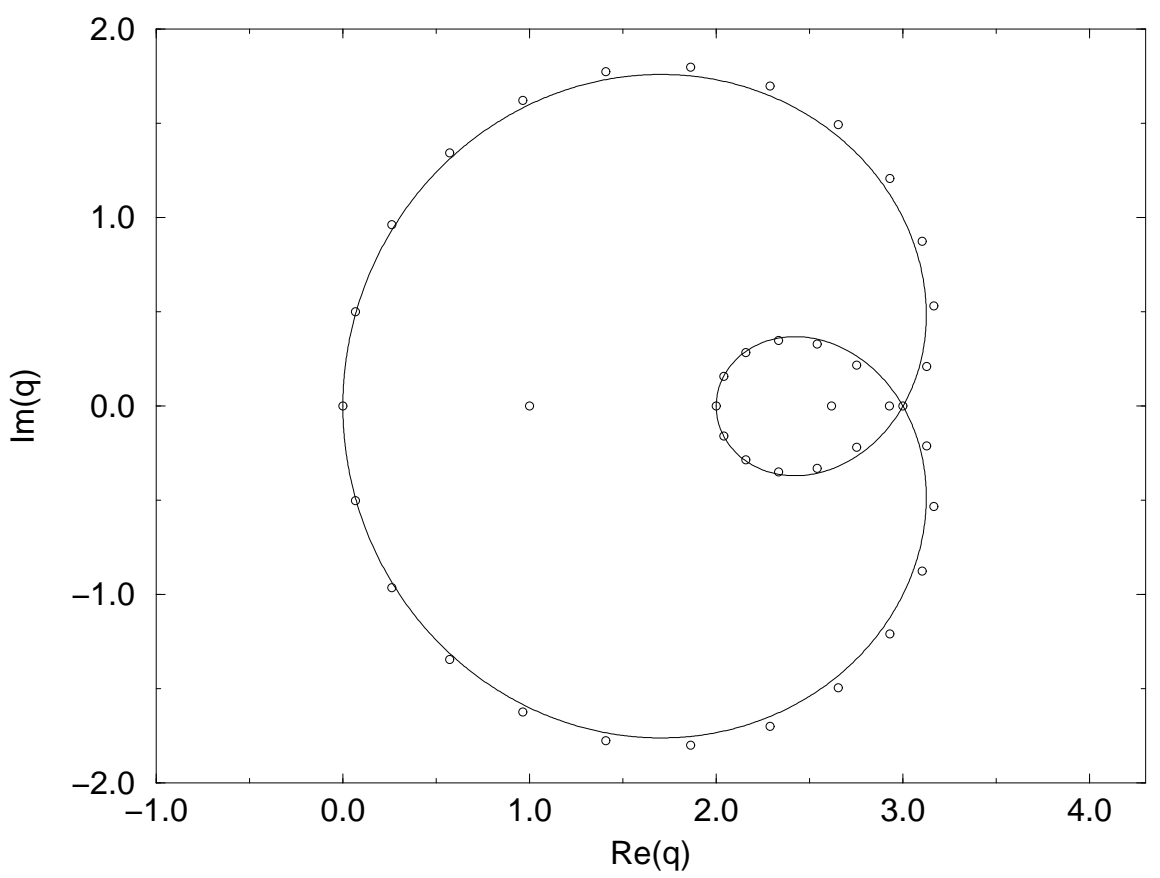

FIG. 3. Locus $\mathcal{B}$ for the function $W$ for a cyclic strip of the triangular lattice with width $L_{y}=2$. Chromatic zeros for $m=20$ ( $n=40$ vertices) are shown for comparison. 
Because the locus $\mathcal{B}$ depends only on $\mathcal{D}$ and is independent of $\mathcal{N}$, it is the same for the cyclic and twisted cyclic strips; hence, in this discussion, we shall group both together and drop the "twisted" label. The locus $\mathcal{B}$ is shown in Fig. 3 and separates the complex $q$ plane into three different regions. We find that

$$
q_{c}\left(t\left(L_{y}=2, c y c .\right)\right)=3
$$

This may be compared with the value of $q_{c}$ for the full $2 \mathrm{D}$ lattice, which is $q_{c}($ tri $)=4$ [11, 7$]$. The region $R_{1}$ contains the real interval $q>3$. The other two regions, $R_{2}$ and $R_{3}$, include the respective real intervals $2<q<3$ and $0<q<2$. We find that the leading $\lambda^{\prime} s$ in regions $R_{1}, R_{2}$ and $R_{3}$ are respectively $\lambda_{\max }=\lambda_{2}, \lambda_{1}$, and $\lambda_{3}$. It follows that

$$
\begin{gathered}
W\left(t\left(L_{y}=2, c y c .\right), q\right)=D_{3}=q-2 \text { for } q \in R_{1} \\
\left|W\left(t\left(L_{y}=2, c y c .\right), q\right)\right|=1 \text { for } q \in R_{2} \\
\left|W\left(t\left(L_{y}=2, c y c .\right), q\right)\right|=\left|\frac{1}{2}[5-2 q+\sqrt{9-4 q}]\right|^{1 / 2} \text { for } q \in R_{3}
\end{gathered}
$$

As an algebraic curve, the boundary $\mathcal{B}$ has a multiple point of index 2 at $q=q_{c}$ in the technical terminology of algebraic geometry [26] (i.e. two branches of the curve cross at this point with two separate tangents). As is clear in Fig. (3), for this strip, $\mathcal{B}$ has no support for $\operatorname{Re}(q)<0$. This is in accord with our earlier inferences noted above, since the global-circuit condition is necessary, but not sufficient, for $\mathcal{B}$ to have support for $\operatorname{Re}(q)<0$.

For the numerator of the generating function, we find, for the cyclic strip,

$$
\begin{gathered}
A_{t, 0}=P\left(K_{4}, q\right)=q(q-1)(q-2)(q-3) \\
A_{t, 1}=q(q-1)(q-2)^{2} \\
A_{t, 2}=-q(q-1)(q-2)^{4}
\end{gathered}
$$

where $P\left(K_{p}, q\right)$ is the chromatic polynomial for the complete graph on $p$ vertices,

$$
P\left(K_{p}, q\right)=\prod_{s=0}^{p-1}(q-s)
$$

For the twisted cyclic strip (for which $\operatorname{deg}_{x}(\mathcal{N})=0$ ) we find 


$$
A_{t t, 0}=A_{t, 0}=P\left(K_{4}, q\right)
$$

By the same methods as described above, we obtain

$$
\begin{gathered}
P\left(t\left(L_{y}=2, \text { cyc. }\right)_{m}, q\right)=q^{2}-3 q+1+\left(\lambda_{t, 2}\right)^{m}+(q-1)\left[\left(\lambda_{t, 3}\right)^{m}+\left(\lambda_{t, 4}\right)^{m}\right] \\
=q^{2}-3 q+1+(q-2)^{2 m}+2^{1-m}(q-1) \sum_{s=0}^{[m / 2]}\left(\begin{array}{c}
m \\
2 s
\end{array}\right)(5-2 q)^{m-2 s}(9-4 q)^{s} \\
P\left(t\left(L_{y}=2, \text { twisted cyc. }\right)_{m}, q\right)=-1+\left(\lambda_{t, 2}\right)^{m}-\frac{(q-1)(q-3)}{\sqrt{9-4 q}}\left[\left(\lambda_{t, 3}\right)^{m}-\left(\lambda_{t, 4}\right)^{m}\right] \\
=-1+(q-2)^{2 m}-2^{1-m}(q-1)(q-3) \sum_{s=0}^{[(m-1) / 2]}\left(\begin{array}{c}
m \\
2 s+1
\end{array}\right)(5-2 q)^{m-2 s-1}(9-4 q)^{s}
\end{gathered}
$$

where in the upper limits on the sums, $[x]$ denotes the integral part of $x$.

\section{ASYMMETRIC HOMEOMORPHIC EXPANSIONS OF THE SQUARE STRIP}

It is also of interest to study homeomorphic expansions of the cyclic square strip. I In Refs. [21] we analyzed such homeomorphic expansions of a square strip with free longitudinal boundary conditions (and free transverse boundary conditions). In particular, let us consider the case in which for a $L_{y}=2$ square strip, we add $k_{1}-2$ vertices to each of the upper horizontal bonds and $k_{2}-2$ vertices to each of the lower horizontal bonds. For $k_{1}=k_{2}$, the resulting $\lambda$ 's are polynomial, and the chromatic polynomials can be written in a relatively simple form [24]. Here we consider the case $k_{1} \neq k_{2}$, which is more complicated. We first comment on a wider set of graphs, defined such that for each square in the original strip one has a choice as to whether to assign the $k_{1}$ vertices to the upper longitudinal side and $k_{2}$ vertices to the lower side, or vice versa. We define a parity vector

$$
\Sigma=\left(\sigma_{1}, \sigma_{2}, \ldots \sigma_{m}\right)
$$

in which the $\sigma_{j}=+$ if for the $j$ 'th $p$-gon, the assignment is (upper,lower) $=\left(k_{1}, k_{2}\right)$ and $\sigma_{j}=-$ if the assignment is (upper,lower) $=\left(k_{2}, k_{1}\right)$. We shall denote this strip graph as

\footnotetext{
${ }^{6}$ A homeomorphic expansion of a graph consists in the addition of degree-2 vertices to bonds of that graph.
} 


$$
(C h)_{k_{1}, k_{2}, \Sigma, c y c ., m}=H E L_{k_{1}-2, k_{2}-2}\left(G_{s q\left(L_{y}=2\right), c y c ., m}\right)
$$

It will be convenient to define the special $\Sigma$ vector

$$
\Sigma_{+, m}=(+,+, \ldots,+)
$$

where the dimension $m$ of $\Sigma$ can be implicit. Obviously, the $(C h)_{k_{1}, k_{2}, \Sigma, c y c ., m}$ graphs are invariant under a reflection about the longitudinal axis, which in the $k_{1} \neq k_{2}$ case amounts to a simultaneous reversal of the signs of all of the $\sigma_{j}$ 's. Let us define this parity operation on $\Sigma$ as

$$
P(\Sigma)=-\Sigma
$$

Then

$$
(C h)_{k_{1}, k_{2}, \Sigma, c y c, m}=(C h)_{k_{1}, k_{2}, P(\Sigma), c y c,, m}=(C h)_{k_{2}, k_{1}, \Sigma, c y c,, m}
$$

The total number of vertices is

$$
v\left((C h)_{k_{1}, k_{2}, \Sigma, c y c ., m}\right)=(p-2) m
$$

independent of $\Sigma$, where

$$
p=k_{1}+k_{2}
$$

For the strip with free longitudinal boundary conditions, we showed in Ref. [21] that the chromatic polynomial $P\left((C h)_{k_{1}, k_{2}, \Sigma, m}, q\right)$ was (i) independent of $\Sigma$ and (ii) depended only on the homeomorphic expansion indices $k_{1}$ and $k_{2}$ through their sum, $p$. However, these properties do not hold for the cyclic strip $(C h)_{k_{1}, k_{2}, \Sigma, c y c, m}$. This can be understood as a consequence of the fact that tying together the ends via the periodic longitudinal boundary conditions increases the constraints on the coloring of the graphs.

To study asymmetric homeomorphic expansions, we shall consider the case $\left(k_{1}, k_{2}\right)=$ $(k, 2)$ (whence $p=k+2$ ) with $\Sigma=\Sigma_{+, m}$, i.e., the family

$$
(C h)_{k_{1}=k, k_{2}=2, \Sigma_{+, m}, c y c ., m}
$$

For this calculation we use a generating function of the form

$$
\Gamma\left((C h)_{k, 2, \Sigma_{+}, c y c .}, q, x\right)=\sum_{m=2}^{\infty} x^{m-2} P\left((C h)_{k, 2, \Sigma_{+}, m, c y c, m}, q\right)
$$


From eq. (6.6), the total number of vertices is $n=\mathrm{km}$. We obtain a generating function of the form given by eq. (2.3) with (2.4) and (2.5), where $\operatorname{deg}_{x}(\mathcal{N})=3$ and $\operatorname{deg}_{x}(\mathcal{D})=5$. The denominator of the generating function is

$$
\begin{aligned}
\mathcal{D}= & {\left[1-(-1)^{k} x\right]\left(1-D_{k+2} x\right)\left[1+(-1)^{k}(q-2) x\right] \times } \\
& {\left[1+\left[D_{k+1}+(-1)^{k}(q-2)\right] x+\left[D_{k+1}(-1)^{k}(q-2)-(-1)^{k} D_{k}\right] x^{2}\right] }
\end{aligned}
$$

We thus have

$$
\begin{gathered}
\lambda_{h, 1}=(-1)^{k} \\
\lambda_{h, 2}=D_{k+2} \\
\lambda_{h, 3}=-(-1)^{k}(q-2) \\
\lambda_{h,(4,5)}=\frac{1}{2}\left[-D_{k+1}-(-1)^{k}(q-2) \pm\left(\left[D_{k+1}-(-1)^{k}(q-2)\right]^{2}+4 D_{k}(-1)^{k}\right)^{1 / 2}\right]
\end{gathered}
$$

(where the subscript $h$ means homeomorphic.) For the chromatic polynomial we obtain

$P\left((C h)_{k, 2, \Sigma_{+}, m, c y c, m}, q\right)=\left(q^{2}-3 q+1\right)(-1)^{k m}+\left(\lambda_{h, 2}\right)^{m}+(q-1)\left[\left(\lambda_{h, 4}\right)^{m}+\left(\lambda_{h, 5}\right)^{m}\right]$

Note that $\lambda_{h, 3}$ does not occur in $P$. For illustration, the coefficient functions entering in the numerator $\mathcal{N}$ are given in the Appendix for $k=3,4$.

Fig. 4 shows the analytic structure of the $W$ function for a cyclic strip of the square lattice with homeomorphic expansion parameters $\left(k_{1}, k_{2}\right)=(\mathrm{a})(2,3)(\mathrm{b})(2,4)$. In these cases we find the respective values (a) $q_{c} \simeq 2.455$ and (b) $q_{c}=2$. In the region $R_{1}$ the leading term is $\lambda_{h, 2}$. In these cases the boundaries $\mathcal{B}$ do not have any part with $\operatorname{Re}(q)<0$. 

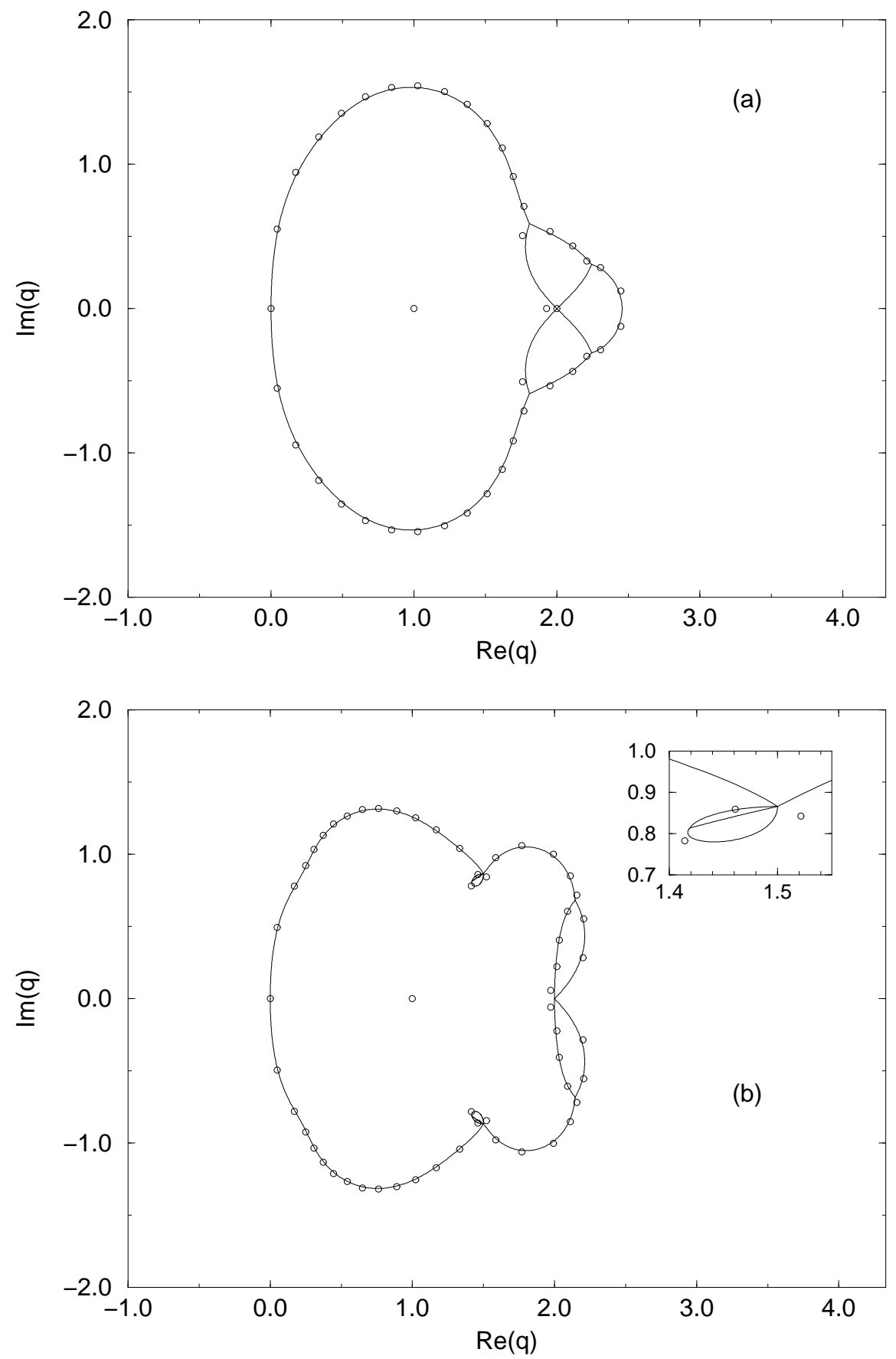

FIG. 4. Boundary $\mathcal{B}$ in the $q$ plane for $W$ function for an asymmetric cyclic chain of (a) 5 -gons $(k=3)$ (b) 6-gons $(k=4)$. Chromatic zeros for $m=14$ are shown for comparison. 


\section{CONCLUSIONS}

In this paper, we have given exact calculations of the zero-temperature partition function of the $q$-state Potts antiferromagnet strips of the square, kagomé and triangular lattices with periodic longitudinal boundary conditions (and free transverse boundary conditions). These yield, in the limit of infinite length, the ground-state entropy $S_{0}=k_{B} \ln W$ via eq. (1.2). These results are of fundamental interest since this model exhibits nonzero ground state entropy $S_{0}>0$ for sufficiently large $q$ and hence is an exception to the third law of thermodynamics. We also include results for homeomorphic expansions of the square lattice strip. The analytic properties of $W(q)$ are determined and related to zeros of the chromatic polynomial for long finite strips.

This research was supported in part by the NSF grant PHY-97-22101. 


\section{APPENDIX}

We list in this appendix the coefficient functions for the numerators of the generating functions for the cyclic strips of the square and kagomé lattices and for asymmetric homeomorphic expansions of the cyclic square strip.

\section{A. Strip of the Square Lattice}

For the cyclic strip of the square lattice with $L_{y}=3$ we calculate

$$
\begin{aligned}
& A_{s q, 0}=q(q-1)\left(q^{2}-3 q+3\right)^{2} \\
& A_{s q, 1}=-2 q(q-1)\left(q^{5}-6 q^{4}+11 q^{3}-21 q+16\right) \\
& A_{s q, 2}=-q(q-1)\left(3 q^{8}-45 q^{7}+306 q^{6}-1242 q^{5}+3301 q^{4}-5854 q^{3}\right. \\
& \left.+6703 q^{2}-4482 q+1318\right) \\
& A_{s q, 3}=-2 q(q-1)\left(q^{10}-24 q^{9}+250 q^{8}-1516 q^{7}+5992 q^{6}-16230 q^{5}\right. \\
& \left.+30580 q^{4}-39552 q^{3}+33530 q^{2}-16764 q+3732\right) \\
& A_{s q, 4}=q(q-1)\left(7 q^{11}-159 q^{10}+1636 q^{9}-10077 q^{8}+41328 q^{7}-118548 q^{6}\right. \\
& \left.+242569 q^{5}-353514 q^{4}+358776 q^{3}-240824 q^{2}+95932 q-17119\right) \\
& A_{s q, 5}=-2 q(q-1)^{2}\left(4 q^{11}-91 q^{10}+935 q^{9}-5728 q^{8}+23249 q^{7}-65620 q^{6}\right. \\
& \left.+131260 q^{5}-185622 q^{4}+181164 q^{3}-115604 q^{2}+43126 q-7070\right) \\
& A_{s q, 6}=q(q-1)^{2}\left(2 q^{12}-49 q^{11}+534 q^{10}-3412 q^{9}+14151 q^{8}-39685 q^{7}\right. \\
& \left.+75440 q^{6}-92942 q^{5}+62238 q^{4}-455 q^{3}-36019 q^{2}+27008 q-6808\right) \\
& A_{s q, 7}=2 q(q-2)(q-3)(q-1)^{4}\left(q^{9}-19 q^{8}+162 q^{7}-818 q^{6}+2706 q^{5}\right.
\end{aligned}
$$




$$
\begin{gathered}
\left.-6090 q^{4}+9318 q^{3}-9330 q^{2}+5535 q-1477\right) \\
A_{s q, 8}=-q(q-4)(q-2)^{3}(q-1)^{5}\left(q^{3}-6 q^{2}+13 q-11\right) \times \\
\left(q^{4}-9 q^{3}+29 q^{2}-40 q+22\right)
\end{gathered}
$$

\section{B. Strip of the Kagomé Lattice}

For our cyclic strip of the Kagomé (kg) lattice we find

$$
\begin{gathered}
A_{k g, 0}=q(q-1)^{2}(q-2)^{2} \\
A_{k g, 1}=-q(q-1)(q-2)^{2}\left(2 q^{5}-17 q^{4}+59 q^{3}-105 q^{2}+104 q-59\right) \\
A_{k g, 2}=q(q-1)(q-2)\left(2 q^{9}-37 q^{8}+311 q^{7}-1547 q^{6}+4994 q^{5}\right. \\
\left.\quad-10845 q^{4}+15932 q^{3}-15484 q^{2}+9250 q-2656\right) \\
A_{k g, 3}=q(q-1)(q-2)\left(3 q^{11}-69 q^{10}+726 q^{9}-4604 q^{8}+19522 q^{7}-58029 q^{6}\right. \\
\left.+123231 q^{5}-186853 q^{4}+198437 q^{3}-141104 q^{2}+60968 q-12292\right) \\
A_{k g, 4}=-q(q-1)^{2}(q-2)^{2}\left(2 q^{11}-34 q^{10}+251 q^{9}-1020 q^{8}+2290 q^{7}-1840 q^{6}-4218 q^{5}\right. \\
\left.+14163 q^{4}-16324 q^{3}+4750 q^{2}+6204 q-4476\right) \\
A_{k g, 5}= \\
q(q-1)^{2}(q-2)^{4}\left(7 q^{10}-121 q^{9}+953 q^{8}-4521 q^{7}+14396 q^{6}-32498 q^{5}\right. \\
\left.+53581 q^{4}-65213 q^{3}+57416 q^{2}-33432 q+9720\right) \\
A_{k g, 7}= \\
\left.+10142 q^{2}-6446 q+2110\right) \\
\left.A_{k g, 6}=-1\right)^{4}(q-2)^{8}\left(5 q^{6}-54 q^{5}+232 q^{4}-511 q^{3}+608 q^{2}-380 q+118\right) \\
q(q-1)^{3}(q-2)^{6}\left(9 q^{8}-129 q^{7}+810 q^{6}-2927 q^{5}+6708 q^{4}-10133 q^{3}\right.
\end{gathered}
$$




\section{Asymmetric Homeomorphic Expansions of the Square Strip}

For the case $k=3$ defined in the text, the coefficients entering in the numerator of the generating function are

$$
\begin{gathered}
A_{k=3,0}=q(q-1)(q-2)\left(q^{3}-4 q^{2}+7 q-5\right) \\
A_{k=3,1}=-q(q-1)(q-2)\left(2 q^{4}-12 q^{3}+27 q^{2}-29 q+13\right) \\
A_{k=3,2}=-q(q-1)^{2}(q-2)^{2}\left(q^{2}-q-1\right) \\
A_{k=3,3}=q(q-2)^{4}(q-1)^{2}\left(q^{2}-2 q+2\right)
\end{gathered}
$$

For the case $k=4$ we find

$$
\begin{gathered}
A_{k=4,0}=q(q-1)\left(q^{6}-8 q^{5}+28 q^{4}-56 q^{3}+70 q^{2}-53 q+19\right) \\
A_{k=4,1}=q(q-1)\left(2 q^{7}-21 q^{6}+94 q^{5}-236 q^{4}+365 q^{3}-356 q^{2}+207 q-56\right) \\
A_{k=4,2}=-q(q-1)^{2}\left(2 q^{6}-17 q^{5}+59 q^{4}-107 q^{3}+107 q^{2}-56 q+11\right) \\
A_{k=4,3}=-q(q-2)(q-1)^{2}\left(q^{3}-5 q^{2}+8 q-5\right)\left(q^{4}-5 q^{3}+10 q^{2}-10 q+5\right)
\end{gathered}
$$

[1] R. B. Potts, Proc. Camb. Phil. Soc. 48 (1952) 106.

[2] F. Y. Wu, Rev. Mod. Phys. 54 (1982) 235; errata, ibid 55 (1983) 315.

[3] M. Aizenman and E. H. Lieb, J. Stat. Phys. 24 (1981) 279.

[4] Y. Chow and F. Y. Wu, Phys. Rev. B36 (1987) 285.

[5] R. C. Read, J. Combin. Theory 4 (1968) 52. 
[6] R. C. Read and W. T. Tutte, "Chromatic Polynomials" in Selected Topics in Graph Theory, 3, (Academic Press, NY, 1988).

[7] R. Shrock and S.-H. Tsai, Phys. Rev. E55 (1997) 5165.

[8] E. H. Lieb, Phys. Rev. 162 (1967) 162.

[9] N. L. Biggs, R. M. Damerell, and D. A. Sands, J. Combin. Theory B 12 (1972) 123; N. L. Biggs and G. H. Meredith, ibid., B 20 (1976) 5.

[10] S. Beraha, J. Kahane, and N. Weiss, J. Combin. Theory B 28 (1980) 52.

[11] R. J. Baxter, J. Phys. A 20 (1987) 5241.

[12] R. C. Read and G. F. Royle, in Graph Theory, Combinatorics, and Applications (Wiley, NY, 1991), vol. 2, p. 1009.

[13] R. C. Read and E. G. Whitehead, Discrete Math. 204 (1999) 337.

[14] R. Shrock and S.-H. Tsai, J. Phys. A 30 (1997) 495.

[15] R. Shrock and S.-H. Tsai, Phys. Rev. E56 (1997) 1342, 4111.

[16] R. Shrock and S.-H. Tsai, Phys. Rev. E56 (1997) 3935.

[17] R. Shrock and S.-H. Tsai, Phys. Rev. E55 (1997) 6791; Phys. Rev. E56 (1997) 2733, 4111.

[18] R. Shrock and S.-H. Tsai, J. Phys. A 31 (1998) 9641.

[19] M. Roček, R. Shrock, and S.-H. Tsai, Physica A252 (1998) 505.

[20] M. Roček, R. Shrock, and S.-H. Tsai, Physica A259 (1998) 367.

[21] R. Shrock and S.-H. Tsai, Physica A259 (1998) 315.

[22] R. Shrock and S.-H. Tsai, Phys. Rev. E58 (1998) 4332. Some obvious misprints in Table 1 are 
corrected in cond-mat/9808057.

[23] R. Shrock and S.-H. Tsai, Physica A265 (1999) 186.

[24] R. Shrock and S.-H. Tsai, J. Phys. A 32 (Lett.) (1999) L195.

[25] R. Shrock and S.-H. Tsai, Phys. Rev. E, in press (ITB-SB-99-11).

[26] R. Hartshorne, Algebraic Geometry (Springer, New York, 1977). 